\title{
AN AX-KOCHEN-ERSHOV THEOREM FOR MONOTONE DIFFERENTIAL-HENSELIAN FIELDS
}

\author{
TIGRAN HAKOBYAN
}

\begin{abstract}
Scanlon 5 proves Ax-Kochen-Ershov type results for differentialhenselian monotone valued differential fields with many constants. We show how to get rid of the condition with many constants.
\end{abstract}

\section{INTRODUCTION}

Let $\boldsymbol{k}$ be a differential field (always of characteristic 0 in this paper, with a single distinguished derivation). Let also an ordered abelian group $\Gamma$ be given. This gives rise to the Hahn field $K=\boldsymbol{k}\left(\left(t^{\Gamma}\right)\right)$, to be considered in the usual way as a valued field. We extend the derivation $\partial$ of $\boldsymbol{k}$ to a derivation on $K$ by

$$
\partial\left(\sum_{\gamma} a_{\gamma} t^{\gamma}\right):=\sum_{\gamma} \partial\left(a_{\gamma}\right) t^{\gamma} .
$$

Scanlon [5] extends the Ax-Kochen-Ershov theorem (see [3, 4]) to this differential setting. This includes requiring that $\boldsymbol{k}$ is linearly surjective in the sense that for each nonzero linear differential operator $A=a_{0}+a_{1} \partial+\cdots+a_{n} \partial^{n}$ over $\boldsymbol{k}$ we have $A(\boldsymbol{k})=\boldsymbol{k}$. Under this assumption, $K$ is differential-henselian (see Section 1 for this notion), and the theory $\operatorname{Th}(K)$ of $K$ as a valued differential field (see also Section 1 for this) is completely axiomatized by:

(1) the axiom that there are many constants;

(2) the theory $\operatorname{Th}(\boldsymbol{k})$ of the differential residue field $\boldsymbol{k}$;

(3) the theory $\operatorname{Th}(\Gamma)$ of the ordered abelian value group;

(4) the axioms for differential-henselian valued fields.

As to (1), having many constants means that every element of the differential field has the same valuation as some element of its constant field. This holds for $K$ as above (whether or not $\boldsymbol{k}$ is linearly surjective) because the constant field of $K$ is $C_{K}=C_{\boldsymbol{k}}\left(\left(t^{\Gamma}\right)\right)$. This axiom plays an important role in some proofs of [5]. Below we drop the "many constants" axiom and generalize the theorem above to a much larger class of differential-henselian valued fields. This involves a more general way of extending the derivation of $\boldsymbol{k}$ to $K$.

In more detail, let $c: \Gamma \rightarrow \boldsymbol{k}$ be an additive map. Then the derivation $\partial$ of $\boldsymbol{k}$ extends to a derivation $\partial_{c}$ of $K$ by setting

$$
\partial_{c}\left(\sum_{\gamma} a_{\gamma} t^{\gamma}\right):=\sum_{\gamma}\left(\partial\left(a_{\gamma}\right)+c(\gamma) a_{\gamma}\right) t^{\gamma} .
$$

Thus $\partial_{c}$ is the unique derivation on $K$ that extends $\partial$, respects infinite sums, and satisfies $\partial_{c}\left(t^{\gamma}\right)=c(\gamma) t^{\gamma}$ for all $\gamma$. The earlier case has $c(\gamma)=0$ for all $\gamma$. Another case is where $\boldsymbol{k}$ contains $\mathbb{R}$ as a subfield, $\Gamma=\mathbb{R}$, and $c: \mathbb{R} \rightarrow \boldsymbol{k}$ is the inclusion map; then $\partial_{c}\left(t^{r}\right)=r t^{r}$ for $r \in \mathbb{R}$. 
Let $K_{c}$ be the valued differential field $K$ with $\partial_{c}$ as its distinguished derivation. Assume in addition that $\boldsymbol{k}$ is linearly surjective. Then $K_{c}$ is differential-henselian, and Scanlon's theorem above generalizes as follows:

Theorem 1. The theory $\operatorname{Th}\left(K_{c}\right)$ is completely determined by $\operatorname{Th}(\boldsymbol{k}, \Gamma ; c)$, where $(\boldsymbol{k}, \Gamma ; c)$ is the 2-sorted structure consisting of the differential field $\boldsymbol{k}$, the ordered abelian group $\Gamma$, and the additive map $c: \Gamma \rightarrow \boldsymbol{k}$.

We actually prove in Section 2 a stronger version with the one-sorted structure $K_{c}$ expanded to a 2-sorted one, with $\Gamma$ as the underlying set for the second sort, and as extra primitives the cross-section $\gamma \mapsto t^{\gamma}: \Gamma \rightarrow K$, the set $\boldsymbol{k} \subseteq K$, and the map $c: \Gamma \rightarrow \boldsymbol{k}$.

The question arises: which complete theories of valued differential fields are covered by Theorem 11? The answer involves the notion of monotonicity: a valued differential field $F$ with valuation $v$ is said to be monotone if $v\left(f^{\prime}\right) \geq v(f)$ for all $f \in F$; as usual, $f^{\prime}$ denotes the derivative of $f \in F$ with respect to the distinguished derivation of $F$. The valued differential fields $K_{c}$ are all clearly monotone. We show:

Theorem 2. Every monotone differential-henselian valued field is elementarily equivalent to some $K_{c}$ as in Theorem 1 .

This is proved in Section 3 and is analogous to the result from [5] that any differentialhenselian valued field with many constants is elementarily equivalent to some $K$ as in Scanlon's theorem stated in the beginning of this Introduction. (In fact, that result follows from the "complete axiomatization" given in that theorem.)

Theorem 2 has a nice algebraic consequence, generalizing [1, Corollary 8.0.2]:

Corollary 1. If a valued differential field $F$ is monotone and differential-henselian, then every valued differential field extension of $F$ that is algebraic over $F$ is also (monotone and) differential-henselian.

See Section 4. To state further results it is convenient to introduce some notation. Let $F$ be a differential field. For nonzero $f \in F$ we set $f^{\dagger}:=f^{\prime} / f$ and $F^{\dagger}:=\left\{f^{\dagger}: f \in F^{\times}\right\}$, where $F^{\times}:=F \backslash\{0\}$.

So far our only assumption on $c: \Gamma \rightarrow k$ is that it is additive, but the case $c(\Gamma) \cap \boldsymbol{k}^{\dagger}=\{0\}$ is of particular interest: it is not hard to show that then the constant field of $K_{c}$ is $C_{\boldsymbol{k}}\left(\left(t^{\Delta}\right)\right)$, where the value group $\Delta$ of the constant field equals $\operatorname{ker}(c)$ and is a pure subgroup of $\Gamma$. Conversely (see Section 3 ):

Theorem 3. Every monotone differential-henselian valued field $F$ such that $v\left(C_{F}^{\times}\right)$ is pure in $v\left(F^{\times}\right)$is elementarily equivalent to some $K_{c}$ as in Theorem 1 with $c(\Gamma) \cap \boldsymbol{k}^{\dagger}=\{0\}$.

The referee showed us an example of a monotone henselian valued differential field $F$ for which $v\left(C_{F}^{\times}\right)$is not pure in $v\left(F^{\times}\right)$. In Section 4 we give an example of a monotone differential-henselian field $F$ such that $v\left(C_{F}^{\times}\right)$is not pure in $v\left(F^{\times}\right)$.

The hypothesis of Theorem 3 that $v\left(C_{F}^{\times}\right)$is pure in $v\left(F^{\times}\right)$holds if the residue field is algebraically closed or real closed (see Section 4). It includes also the case of main interest to us, where $F$ has few constants, that is, the valuation is trivial on $C_{F}$. In that case any $c$ as in Theorem 3 is injective by Corollary 3.2 ,

Section 3 contains examples of additive maps $c: \Gamma \rightarrow \boldsymbol{k}$ for which $K_{c}$ has few constants, including a case where $\operatorname{Th}\left(K_{c}\right)$ is decidable. Two of those examples show 
that in Theorem 1, even when we have few constants, the traditional Ax-KochenErshov principle without the map $c$ does not hold. (It does hold in Scanlon's theorem where $c=0$, but in general we do not expect to have a $c$ that is definable in the valued differential field structure.)

\section{Preliminaries}

Adopting terminology from [1], a valued differential field is a differential field $K$ together with a (Krull) valuation $v: K^{\times} \rightarrow \Gamma$ whose residue field $\mathbf{k}:=\mathcal{O} / \mathcal{O}$ has characteristic zero; here $\Gamma=v\left(K^{\times}\right)$is the value group, and we also let $\mathcal{O}=\mathcal{O}_{K}$ denote the valuation ring of $v$ with maximal ideal $\mathcal{O}$, and let

$$
C=C_{K}:=\left\{f \in K: f^{\prime}=0\right\}
$$

denote the constant field of the differential field $K$. We use notation from [1] for elements $a, b$ of a valued field with valuation $v$ we set

$$
a \asymp b: \Leftrightarrow v a=v b, \quad a \preceq b \Leftrightarrow b \succeq a: \Leftrightarrow v a \geq v b, \quad a \prec b \Leftrightarrow b \succ a: \Leftrightarrow v a>v b .
$$

Let $K$ be a valued differential field as above, and let $\partial$ be its derivation. We say that $K$ has many constants if $v\left(C^{\times}\right)=\Gamma$. We say that the derivation of $K$ is small if $\partial(\mathcal{O}) \subseteq \mathcal{O}$. If $K$, with a small derivation, has many constants, then $K$ is monotone in the sense of [2], that is, $v(f) \leq v\left(f^{\prime}\right)$ for all $f \in K$. We say that $K$ has few constants if $v\left(C^{\times}\right)=\{0\}$. Note: if $K$ is monotone, then its derivation is small; if the derivation of $K$ is small, then $\partial$ is continuous with respect to the valuation topology on $K$. Note also that if $K$ is monotone, then so is any valued differential field extension with small derivation and the same value group as $K$.

From now on we assume that the derivation of $K$ is small. This has the effect (see 2] or [1, Lemma 4.4.2]) that also $\partial(\mathcal{O}) \subseteq \mathcal{O}$, and so $\partial$ induces a derivation on the residue field; we view $\mathbf{k}$ below as equipped with this induced derivation, and refer to it as the differential residue field of $K$.

We say that $K$ is differential-henselian (for short: d-henselian) if every differential polynomial $P \in \mathcal{O}\{Y\}=\mathcal{O}\left[Y, Y^{\prime}, Y^{\prime \prime}, \ldots\right]$ whose reduction $\bar{P} \in \mathbf{k}\{Y\}$ has total degree 1 has a zero in $\mathcal{O}$. (Note that for ordinary polynomials $P \in \mathcal{O}[Y]$ this requirement defines the usual notion of a henselian valued field, that is, a valued field whose valuation ring is henselian as a local ring.)

If $K$ is d-henselian, then its differential residue field is clearly linearly surjective: any linear differential equation $y^{(n)}+a_{n-1} y^{(n-1)}+\cdots+a_{0} y=b$ with coefficients $a_{i}, b \in \mathbf{k}$ has a solution in $\mathbf{k}$. This is a key constraint on our notion of d-henselianity. If $K$ is d-henselian, then $\mathbf{k}$ has a lift to $K$, meaning, a differential subfield of $K$ contained in $\mathcal{O}$ that maps isomorphically onto $\mathbf{k}$ under the canonical map from $\mathcal{O}$ onto k; see [1, 7.1.3]. Other items from [1] that are relevant in this paper are the following differential analogues of Hensel's Lemma and of results due to Ostrowski/Krull/Kaplansky on valued fields:

(DV1) If the derivation of $\mathbf{k}$ is nontrivial, then $K$ has a spherically complete immediate valued differential field extension with small derivation; 1, 6.9.5].

(DV2) If $\mathbf{k}$ is linearly surjective and $K$ is spherically complete, then $K$ is $\mathrm{d}$ henselian; [1, 7.0.2].

(DV3) If $\mathbf{k}$ is linearly surjective and $K$ is monotone, then any two spherically complete immediate monotone valued differential field extensions of $K$ are isomorphic over $K$; [1, 7.4.3]. 
We also need a model-theoretic variant of (DV3):

(DV4) Suppose $\mathbf{k}$ is linearly surjective and $K$ is monotone with $v\left(K^{\times}\right) \neq\{0\}$. Let $K^{\bullet}$ be a spherically complete immediate valued differential field extension of $K$. Then $K^{\bullet}$ can be embedded over $K$ into any $\left|v\left(K^{\times}\right)\right|^{+}$-saturated d-henselian monotone valued differential field extension of $K$; [1, 7.4.5].

\section{ELEMENTARY EQUIVALENCE OF MONOTONE DIFFERENTIAL-HENSELIAN FIELDS}

In this section we obtain Theorem 1 from the introduction as a consequence of a more precise result in a 2 -sorted setting. We consider 2 -sorted structures

$$
\mathcal{K}=(K, \Gamma ; v, s, c),
$$

where $K$ is a differential field equipped with a differential subfield $\boldsymbol{k}$ (singled out by a unary predicate symbol), $\Gamma$ is an ordered abelian group, $v: K^{\times} \rightarrow \Gamma=v\left(K^{\times}\right)$is a valuation that makes $K$ into a monotone valued differential field such that $\boldsymbol{k} \subseteq K$ is a lift of the differential residue field, $s: \Gamma \rightarrow K^{\times}$is a cross-section of $v$ (that is, $s$ is a group morphism and $\left.v \circ s=\mathrm{id}_{\Gamma}\right)$, and $c: \Gamma \rightarrow \boldsymbol{k}$ satisfies $c(\gamma)=s(\gamma)^{\dagger}$ for all $\gamma \in \Gamma$ (so $c$ is additive). We construe these $\mathcal{K}$ as $L_{2}$-structures for a natural 2 -sorted language $L_{2}$ (with unary function symbols for $v, s$, and $c$ ). We have an obvious set $\operatorname{Mo}(\ell, s, c)$ of $L_{2}$-sentences whose models are exactly these $\mathcal{K}$; the " $\ell$ " is to indicate the presence of a lift.

For example, for $K=\boldsymbol{k}\left(\left(t^{\Gamma}\right)\right)$ as in the introduction and additive $c: \Gamma \rightarrow \boldsymbol{k}$ we consider $K_{c}$ as a model of $\operatorname{Mo}(\ell, s, c)$ in the obvious way by taking $\boldsymbol{k} \subseteq K$ as lift, and $\gamma \mapsto t^{\gamma}$ as cross-section.

Theorem 2.1. If $\mathcal{K}$ is d-henselian, then $\operatorname{Th}(\mathcal{K})$ is axiomatized by:

(1) $\operatorname{Mo}(\ell, s, c)$;

(2) the axioms for d-henselianity;

(3) $\operatorname{Th}(\boldsymbol{k}, \Gamma ; c)$ with $\boldsymbol{k}$ as differential field and $\Gamma$ as ordered abelian group.

We first develop the required technical material, and give the proof of this theorem at the end of this section. Until further notice, $\mathcal{K}=(K, \Gamma ; \boldsymbol{k}, v, s, c) \models \operatorname{Mo}(\ell, s, c)$. For any subfield $E$ of $K$ we set $\Gamma_{E}:=v\left(E^{\times}\right)$.

We define a good subfield of $\mathcal{K}$ to be a differential subfield of $K$ such that (i) $\boldsymbol{k} \subseteq E$, (ii) $s\left(\Gamma_{E}\right) \subseteq E$, and (iii) $\left|\Gamma_{E}\right| \leq \aleph_{0}$. Thus $\boldsymbol{k}$ is a good subfield of $\mathcal{K}$.

Lemma 2.2. Let $E$ be a good subfield of $\mathcal{K}$ and $x \in K \backslash E$. Then $\left|\Gamma_{E(x)}\right| \leq \aleph_{0}$.

This is well-known; see for example [1, Lemma 3.1.10].

Lemma 2.3. Let $E \subseteq K$ be a good subfield of $\mathcal{K}$ and $\gamma \in \Gamma \backslash \Gamma_{E}$, that is, $s(\gamma) \notin E$. Then $E(s(\gamma))$ is also a good subfield of $\mathcal{K}$.

Proof. From $c(\gamma) \in \boldsymbol{k} \subseteq E$ and $s(\gamma)^{\prime}=c(\gamma) s(\gamma)$ we get that $E(s(\gamma))$ is a differential subfield of $K$ and that condition (i) for being a good subfield is satisfied by $E(s(\gamma))$. For condition (ii) we distinguish two cases:

(1) $n \gamma \in \Gamma_{E}$ for some $n \in \mathbb{N} \geq 1$. Take $n \geq 1$ minimal with $n \gamma \in \Gamma_{E}$. Then $0, \gamma, 2 \gamma, \ldots,(n-1) \gamma$ are in different cosets of $\Gamma_{E}$, so for every $q(X) \in E[X]^{\neq}$of degree $<n$ we get $q(s(\gamma)) \neq 0$. Hence the minimum polynomial of $s(\gamma)$ over $E$ is $X^{n}-s(n \gamma)$. Thus, given any $x \in E(s(\gamma))^{\times}$, we have

$$
x=q_{0}+q_{1} s(\gamma)+\ldots+q_{n-1} s(\gamma)^{n-1}
$$


with $q_{0}, \ldots, q_{n-1} \in E$, not all 0 , so $v(x)=\min _{i=0, \ldots, n-1}\left\{v\left(q_{i}\right)+i \gamma\right\}$. Therefore, $\Gamma_{E(s(\gamma))}=\Gamma_{E}+\mathbb{Z} \gamma$ and hence $s\left(\Gamma_{E(s(\gamma))}\right) \subseteq s\left(\Gamma_{E}\right) \cdot s(\gamma)^{\mathbb{Z}} \subseteq E(s(\gamma))$.

(2) $n \gamma \notin \Gamma_{E}$ for all $n \in \mathbb{N} \geq 1$. Then $0, \gamma, 2 \gamma, \ldots$ are in different cosets of $\Gamma_{E}$, so $s(\gamma)$ is transcendental over $E$ and for any polynomial $q(X)=q_{0}+q_{1} X+\ldots+q_{n} X^{n} \in E[X]$, we have $v(q(s(\gamma)))=\min _{i=0 \ldots, n}\left\{v\left(q_{i}\right)+i \gamma\right\}$. As in case (1) this yields $\Gamma_{E(s(\gamma))}=$ $\Gamma_{E}+\mathbb{Z} \gamma$ and so $s\left(\Gamma_{E(s(\gamma))}\right) \subseteq s\left(\Gamma_{E}\right) \cdot s(\gamma)^{\mathbb{Z}} \subseteq E(s(\gamma))$.

Thus condition (ii) of good subfields holds for $E(s(\gamma))$. Condition (iii) is satisfied by Lemma 2.2 ,

In the rest of this section we fix a d-henselian $\mathcal{K}$. Let $T_{\mathcal{K}}$ be the $L_{2}$-theory given by (1)-(3) in Theorem 2.1. Assume CH (the Continuum Hypothesis), and let

$$
\mathcal{K}_{1}=\left(K_{1}, \Gamma_{1} ; v_{1}, s_{1}, c_{1}\right), \quad \mathcal{K}_{2}=\left(K_{2}, \Gamma_{2} ; v_{2}, s_{2}, c_{2}\right)
$$

be saturated models of $T_{\mathcal{K}}$ of cardinality $\aleph_{1}$; remarks following Corollary 2.6 explain why we can assume $\mathrm{CH}$. Then the structures $\left(\boldsymbol{k}_{1}, \Gamma_{1} ; c_{1}\right)$ and $\left(\boldsymbol{k}_{2}, \Gamma_{2} ; c_{2}\right)$ are also saturated of cardinality $\aleph_{1}$, where $\boldsymbol{k}_{1}$ and $\boldsymbol{k}_{2}$ are the lifts of the differential residue fields of $K_{1}$ and $K_{2}$ respectively. Since $\left(\boldsymbol{k}_{1}, \Gamma_{1} ; c_{1}\right)$ and $\left(\boldsymbol{k}_{2}, \Gamma_{2} ; c_{2}\right)$ are elementarily equivalent to $(\boldsymbol{k}, \Gamma ; c)$, we have an isomorphism $f=\left(f_{r}, f_{v}\right)$ from $\left(\boldsymbol{k}_{1}, \Gamma_{1} ; c_{1}\right)$ onto $\left(\boldsymbol{k}_{2}, \Gamma_{2} ; c_{2}\right)$ with $f_{r}: \boldsymbol{k}_{1} \rightarrow \boldsymbol{k}_{2}$ and $f_{v}: \Gamma_{1} \rightarrow \Gamma_{2}$.

A map $g: E_{1} \rightarrow E_{2}$ between good subfields $E_{1}$ and $E_{2}$ of $\mathcal{K}_{1}$ and $\mathcal{K}_{2}$ respectively, will be called good if

(1) $g: E_{1} \rightarrow E_{2}$ is a differential field isomorphism,

(2) $g$ extends $f_{r}$,

(3) $f_{v} \circ v_{1}=v_{2} \circ g$

(4) $g \circ s_{1}=s_{2} \circ f_{v}$.

Note that then $g$ is also an isomorphism of the valued subfield $E_{1}$ of $K_{1}$ onto the valued subfield $E_{2}$ of $K_{2}$. The map $f_{r}: \boldsymbol{k}_{1} \rightarrow \boldsymbol{k}_{2}$ is clearly a good map.

Proposition 2.4. $\mathcal{K}_{1} \cong \mathcal{K}_{2}$.

Proof. We claim that the collection of good maps is a back-and-forth system between $K_{1}$ and $K_{2}$. (By the saturation assumption this yields the desired result.) This claim holds trivially if $\Gamma_{1}=\{0\}$, so assume $\Gamma_{1} \neq\{0\}$, and thus $\Gamma_{2} \neq\{0\}$.

Let $g: E_{1} \rightarrow E_{2}$ be a good map and $\gamma \in \Gamma_{1} \backslash \Gamma_{E_{1}}$. By Lemma 2.3 we have good subfields $E_{1}\left(s_{1}(\gamma)\right)$ of $\mathcal{K}_{1}$ and $E_{2}\left(s_{2}\left(f_{v}(\gamma)\right)\right)$ of $\mathcal{K}_{2}$. The proof of that lemma then yields easily a good map

$$
g_{\gamma}: E_{1}\left(s_{1}(\gamma)\right) \rightarrow E_{2}\left(s_{2}\left(f_{v}(\gamma)\right)\right)
$$

that extends $g$ with $g_{\gamma}\left(s_{1}(\gamma)\right)=s_{2}\left(f_{v}(\gamma)\right)$.

Let $g: E_{1} \rightarrow E_{2}$ be a good map and $x \in K_{1} \backslash E_{1}$. We show how to extend $g$ to a good map with $x$ in its domain.

By condition (i) of being a good subfield, $E_{1} \supseteq \boldsymbol{k}_{1}$ and $E_{2} \supseteq \boldsymbol{k}_{2}$. The group $\Gamma_{E_{1}\langle x\rangle}$ is countable by Lemma 2.2. Thus by applying iteratively the construction above to elements $\gamma \in \Gamma_{E_{1}\langle x\rangle}$, we can extend $g$ to a good map $g^{1}: E_{1}^{1} \rightarrow E_{2}^{1}$ with $\Gamma_{E_{1}^{1}}=\Gamma_{E_{1}\langle x\rangle}$. Likewise we can extend $g^{1}$ to a good map $g^{2}: E_{1}^{2} \rightarrow E_{2}^{2}$ with $\Gamma_{E_{1}^{2}}=\Gamma_{E_{1}^{1}\langle x\rangle}$. Iterating this process and taking the union $E_{i}^{\infty}=\bigcup_{n} E_{i}^{n}$, for $i=1,2$, we get a good map $g^{\infty}: E_{1}^{\infty} \rightarrow E_{2}^{\infty}$ extending $g$ such that $\Gamma_{E_{1}^{\infty}} \stackrel{n}{=} \Gamma_{E_{1}^{\infty}\langle x\rangle}$, 
so the valued differential field extension $E_{1}^{\infty}\langle x\rangle$ of $E_{1}^{\infty}$ is immediate. By (DV1) and (DV4) we have a spherically complete immediate valued differential field extension $E_{1}^{\bullet} \subseteq K_{1}$ of $E_{1}^{\infty}\langle x\rangle$. Note that then $E_{1}^{\bullet}$ is also a spherically complete immediate valued differential field extension of $E_{1}^{\infty}$. Likewise we have a spherically complete immediate valued differential field extension $E_{2}^{\bullet} \subseteq K_{2}$ of $E_{2}^{\infty}$. By (DV3) we can extend $g^{\infty}$ to a valued differential field isomorphism $g^{\bullet}: E_{1}^{\bullet} \rightarrow E_{2}^{\bullet}$. It is clear that then $g^{\bullet}$ is a good map extending $g$ with $x$ in its domain.

This finishes the proof of the forth part. The back part is done likewise.

Proof of Theorem 2.1. We can assume the Continuum Hypothesis $(\mathrm{CH})$ for this argument. (This is explained further in the remarks following Corollary 2.6.) Our job is to show that the theory $T_{\mathcal{K}}$ is complete. In other words, given any two models of $T_{\mathcal{K}}$ we need to show they are elementarily equivalent. Using $\mathrm{CH}$ we can assume that these models are saturated of cardinality $\aleph_{1}$, and so they are indeed isomorphic by Proposition 2.4

Note that Theorem 1 is a consequence of Theorem 2.1.

Corollary 2.5. Suppose $\mathcal{K}_{1}=\left(K_{1}, \Gamma_{1} ; v_{1}, s_{1}, c_{1}\right)$ and $\mathcal{K}_{2}=\left(K_{2}, \Gamma_{2} ; v_{2}, s_{2}, c_{2}\right)$ are d-henselian models of $\operatorname{Mo}(\ell, c, s)$. Then: $\mathcal{K}_{1} \equiv \mathcal{K}_{2} \Longleftrightarrow\left(\boldsymbol{k}_{1}, \Gamma_{1} ; c_{1}\right) \equiv\left(\boldsymbol{k}_{2}, \Gamma_{2} ; c_{2}\right)$.

In connection with eliminating the use of $\mathrm{CH}$ we introduce the $L_{2}$-theory $T$ whose models are the d-henselian models of $\operatorname{Mo}(\ell, s, c)$. The structures $(\boldsymbol{k}, \Gamma ; c)$ where $\boldsymbol{k}$ is a differential field, $\Gamma$ is an ordered abelian group, and $c: \Gamma \rightarrow \boldsymbol{k}$, are $L_{c}$-structures for a certain sublanguage $L_{c}$ of $L_{2}$. Now Corollary 2.5 yields:

Corollary 2.6. Every $L_{2}$-sentence is $T$-equivalent to some $L_{c}$-sentence.

The above proof of Corollary 2.6 depends on $\mathrm{CH}$, but $T$ has an explicit axiomatization and so the statement of this corollary is "arithmetic". Therefore this proof can be converted to one using just $\mathrm{ZFC}$ (without $\mathrm{CH}$ ). Thus as an obvious consequence of Corollary 2.6, Theorem 2.1 also holds without assuming $\mathrm{CH}$.

\section{EXISTENCE OF $\boldsymbol{k}, s, c$}

In this section we construct under certain conditions a lift $\boldsymbol{k}$, a cross-section $s$, and a map $c$ as in the previous section.

Proposition 3.1. Assume $\mathcal{K}=(K, \Gamma ; v, s, c) \models \operatorname{Mo}(\ell, c, s)$. Then

$$
\begin{gathered}
s(\operatorname{ker}(c))=C^{\times} \cap s(\Gamma) \quad\left(s o \operatorname{ker}(c) \subseteq v\left(C^{\times}\right)\right), \quad c\left(v\left(C^{\times}\right)\right) \subseteq \boldsymbol{k}^{\dagger}, \\
c(\Gamma) \cap \boldsymbol{k}^{\dagger}=\{0\} \Longleftrightarrow \operatorname{ker}(c)=v\left(C^{\times}\right) .
\end{gathered}
$$

Proof. Let $\gamma \in \Gamma$. If $c(\gamma)=0$, then $s(\gamma)^{\dagger}=0$, so $s(\gamma) \in C^{\times} \cap s(\Gamma)$. If $s(\gamma) \in C^{\times}$, then $c(\gamma)=s(\gamma)^{\dagger}=0$, so $\gamma \in \operatorname{ker}(c)$. This proves the first equality. Next, for the inclusion $c\left(v\left(C^{\times}\right)\right) \subseteq \boldsymbol{k}^{\dagger}$, suppose $\gamma=v a$ with $a \in C^{\times}$. Then $s(\gamma)=u a$ with $u \asymp 1$ in $K$, so $u=d(1+\epsilon)$ with $d \in \boldsymbol{k}^{\times}$and $\epsilon \prec 1$. Hence

$$
c(\gamma)=s(\gamma)^{\dagger}=u^{\dagger}=d^{\dagger}+(1+\epsilon)^{\dagger}=d^{\dagger}+\frac{\epsilon^{\prime}}{1+\epsilon} .
$$

Since $c(\gamma), d^{\dagger} \in \boldsymbol{k}$ and $\epsilon^{\prime} \prec 1$, this gives $\epsilon^{\prime}=0$, so $c(\gamma) \in \boldsymbol{k}^{\dagger}$, as claimed. As to the equivalence, suppose $c(\Gamma) \cap \boldsymbol{k}^{\dagger}=\{0\}$. Then $c\left(v\left(C^{\times}\right)\right)=\{0\}$ by the inclusion that we just proved, so $v\left(C^{\times}\right) \subseteq \operatorname{ker}(c)$. We already have the reverse inclusion, 
so $\operatorname{ker}(c)=v\left(C^{\times}\right)$. For the converse, assume $\operatorname{ker}(c)=v\left(C^{\times}\right)$. Let $\gamma \in \Gamma$ be such that $c(\gamma)=d^{\dagger}$ with $d \in \boldsymbol{k}^{\times}$. Then $s(\gamma)^{\dagger}=d^{\dagger}$, so $s(\gamma) / d \in C^{\times}$, hence $\gamma=v(s(\gamma) / d) \in v\left(C^{\times}\right)$, and thus $c(\gamma)=0$, as claimed.

Examples where $c(\Gamma) \cap \boldsymbol{k}^{\dagger} \neq\{0\}$ : Take any differential field $\boldsymbol{k}$ with $\boldsymbol{k} \neq C_{\boldsymbol{k}}$, and take $\Gamma=\mathbb{Z}$. Then $\boldsymbol{k}^{\dagger} \neq\{0\}$; take any nonzero element $u \in \boldsymbol{k}^{\dagger}$. Then for the additive map $c: \Gamma \rightarrow \boldsymbol{k}$ given by $c(1)=u$ we have $c(\Gamma)=\mathbb{Z} u \subseteq \boldsymbol{k}^{\dagger}$, and so $\boldsymbol{k}\left(\left(t^{\Gamma}\right)\right)_{c}$ is a model of $\operatorname{Mo}(\ell, c, s)$ with $c(\Gamma) \cap \boldsymbol{k}^{\dagger} \neq\{0\}$. By taking $\boldsymbol{k}$ to be linearly surjective, this model is d-henselian.

An example where $c(\Gamma) \cap \boldsymbol{k}^{\dagger}=\{0\}$ : Take $\boldsymbol{k}=\mathbb{T}_{\text {log }}$, the differential field of logarithmic transseries; see [1, Chapter 15 and Appendix A] about $\mathbb{T}_{\log }$, especially the fact that $\mathbb{T}_{\log }$ is linearly surjective. Also $\mathbb{T}_{\log }$ contains $\mathbb{R}$ as a subfield, and $f^{\dagger} \notin \mathbb{R}$ for all nonzero $f \in \mathbb{T}_{\log }$. Next, take $\Gamma=\mathbb{R}$ and define $c: \Gamma \rightarrow \boldsymbol{k}$ by $c(r)=r$. Then $K:=\boldsymbol{k}\left(\left(t^{\Gamma}\right)\right)$ yields a d-henselian model $K_{c}$ of $\operatorname{Mo}(\ell, c, s)$ with $c(\Gamma) \cap \boldsymbol{k}^{\dagger}=\{0\}$. Allen Gehret conjectured an axiomatization of $\operatorname{Th}\left(\mathbb{T}_{\log }\right)$ that would imply its decidability, and thus the decidability of the theory of $K_{c}$. This $K_{c}$ has few constants by the following obvious consequence of Proposition 3.1 .

Corollary 3.2. Suppose $\mathcal{K}=(K, \Gamma ; v, s, c) \models \operatorname{Mo}(\ell, c, s)$. Then:

$c$ is injective and $c(\Gamma) \cap \boldsymbol{k}^{\dagger}=\{0\} \Longleftrightarrow \mathcal{K}$ has few constants.

We now provide an example to show that in Theorem 1 we cannot drop the map $c$ in the case of few constants. Take $\boldsymbol{k}=\mathbb{T}_{\log }$ and $\Gamma=\mathbb{Z}$. Define the additive maps $c_{1}: \Gamma \rightarrow \boldsymbol{k}$ by $c_{1}(1)=1$ and $c_{2}: \Gamma \rightarrow \boldsymbol{k}$ by $c_{2}(1)=\sqrt{2}$; instead of $\sqrt{2}$, any irrational real number will do. Let $K_{1}:=\boldsymbol{k}\left(\left(t^{\Gamma}\right)\right)$ and $K_{2}:=\boldsymbol{k}\left(\left(t^{\Gamma}\right)\right)$ be the differential Hahn fields with derivations defined as in the introduction using the maps $c_{1}$ and $c_{2}$, respectively. They are d-henselian monotone valued differential fields. As in the previous example they have few constants by Corollary 3.2. We claim that $K_{1}$ and $K_{2}$ are not elementarily equivalent as valued differential fields (without $c_{1}$ and $c_{2}$ as primitives), so the traditional Ax-Kochen-Ershov principle does not hold. In $K_{1}$, we have $t^{\dagger}=c(1)=1$ and so $K_{1} \models \exists a \neq 0\left(a^{\dagger}=1\right)$. We now show that $K_{2} \not \models \exists a \neq 0\left(a^{\dagger}=1\right)$. Towards a contradiction, assume $a \in K_{2}^{\times}$is such that $a^{\dagger}=1$. Then $a=t^{k} d(1+\epsilon)$ with $k \in \mathbb{Z}, d \in \boldsymbol{k}^{\times}$and $\epsilon \in K_{2}$ with $\epsilon \prec 1$. Hence $a^{\dagger}=c_{2}(k)+d^{\dagger}+(1+\epsilon)^{\dagger}$, so

$$
k \sqrt{2}+d^{\dagger}+\frac{\epsilon^{\prime}}{1+\epsilon}=1 .
$$

Since $\epsilon^{\prime} \prec 1$ we get $k \sqrt{2}+d^{\dagger}=1$ and $\epsilon^{\prime}=0$. Thus $d^{\dagger}=1-k \sqrt{2} \in \mathbb{R}$. Since $1-k \sqrt{2} \neq 0$, this contradicts $\mathbb{T}_{\log }^{\dagger} \cap \mathbb{R}=\{0\}$.

Next we give an example of a decidable d-henselian monotone valued differential field with few constants. The valued differential field $\mathbb{T}$ of transseries is linearly surjective by [1, Corollary 15.0.2] and [1, Corollary 14.2.2]. As $\mathbb{T}[\mathrm{i}]$ with $\mathrm{i}^{2}=-1$ is algebraic over $\mathbb{T}$, it is also linearly surjective by [1, Corollary 5.4.3]. The proof of [1, Proposition 10.7.10] gives $\left(\mathbb{T}[\mathrm{i}]^{\times}\right)^{\dagger}=\mathbb{T}+\mathrm{i} \partial \mathcal{O}$, where $\mathcal{O}$ is the maximal ideal of the valuation ring of $\mathbb{T}$. Thus taking $\boldsymbol{k}=\mathbb{T}[\mathrm{i}], \Gamma=\mathbb{R}$ and the additive map $c: \Gamma \rightarrow \boldsymbol{k}$ given by $c(r)=\mathrm{i} r$, we have $c(\Gamma) \cap \boldsymbol{k}^{\dagger}=\mathrm{i} \mathbb{R} \cap(\mathbb{T}+\mathrm{i} \partial \mathcal{O})=\{0\}$ and therefore $K:=\mathbb{T}[\mathrm{i}]\left(\left(t^{\mathbb{R}}\right)\right)_{c}$ will be a monotone d-henselian valued differential field with few constants by Corollary 3.2. Moreover, $\operatorname{Th}(K)$ is decidable by Theorem 1, since the 
2 -sorted structure $(\mathbb{T}[\mathrm{i}], \mathbb{R} ; c)$ is interpretable in the valued differential field $\mathbb{T}$ and the latter has decidable theory by [1, Corollary 16.6.3].

In what follows we fix a differential field $K$ with a valuation $v: K^{\times} \rightarrow \Gamma=v\left(K^{\times}\right)$ such that $(K, \Gamma ; v)$ is a monotone valued differential field.

Lemma 3.3. Suppose $(K, \Gamma ; v)$ is d-henselian and $\boldsymbol{k}$ is a lift of its differential residue field. Then $G:=\left\{a \in K^{\times}: a^{\dagger} \in \boldsymbol{k}\right\}$ is a subgroup of $K^{\times}$with $v(G)=\Gamma$.

Proof. Using $(a / b)^{\dagger}=a^{\dagger}-b^{\dagger}$ for $a, b \in K^{\times}$we see that $G$ is a subgroup of $K^{\times}$. Let $\gamma \in \Gamma$; our goal is to find a $g \in G$ with $v g=\gamma$. Take $f \in K^{\times}$with $v f=\gamma$. If $f^{\prime} \prec f$, then [1, 7.1.10] gives $g \in C^{\times}$such that $f \asymp g$, so $g \in G$ and $v g=\gamma$. Next, suppose $f^{\prime} \asymp f$. Then $f^{\dagger} \asymp 1$, so $f^{\dagger}=a+\epsilon$ with $a \in \boldsymbol{k}$ and $\epsilon \in \mathcal{O}$. By [1, Corollary 7.1.9] we have $\mathcal{O}=(1+\mathcal{O})^{\dagger}$, so $\epsilon=(1+\delta)^{\dagger}$ with $\delta \in \mathcal{O}$. Then $\left(\frac{f}{1+\delta}\right)^{\dagger}=a \in \boldsymbol{k}$, so $\frac{f}{1+\delta} \in G$ and $v\left(\frac{f}{1+\delta}\right)=\gamma$.

Recall that if $(K, \Gamma ; v)$ is d-henselian, then a lift of the differential residue field exists. Below we assume a lift $\boldsymbol{k}$ of the differential residue field is given, and we consider the 2 -sorted structure $((K, \boldsymbol{k}), \Gamma ; v)$ (so $\boldsymbol{k}$ is a distinguished subset of $K$ ).

Lemma 3.4. Suppose $((K, \boldsymbol{k}), \Gamma ; v)$ is d-henselian, $\aleph_{1}$-saturated and $G$ is a definable subgroup of $K^{\times}$such that $v(G)=\Gamma$. Then there exists a cross-section $s: \Gamma \rightarrow K^{\times}$such that $s(\Gamma) \subseteq G$.

Proof. First note that $H:=\mathcal{O}^{\times} \cap G$ is a pure subgroup of $G$. The inclusion $H \rightarrow G$ and the restriction of the valuation $v$ to $G$ yield an exact sequence

$$
1 \rightarrow H \rightarrow G \rightarrow \Gamma \rightarrow 0
$$

of abelian groups. Since $H$ is $\aleph_{1}$-saturated as an abelian group, this exact sequence splits; see [1, Corollary 3.3.37]. This yields a cross-section $s: \Gamma \rightarrow K^{\times}$with $s(\Gamma) \subseteq G$.

Combining the previous two lemmas gives us the main result of this section:

Theorem 3.5. Suppose $((K, \boldsymbol{k}), \Gamma ; v)$ is $\mathrm{d}$-henselian and $\aleph_{1}$-saturated. Then there is a cross-section $s: \Gamma \rightarrow K^{\times}$and an additive map $c: \Gamma \rightarrow \boldsymbol{k}$ with $s(\gamma)^{\dagger}=c(\gamma)$ for all $\gamma \in \Gamma$.

Proof. Since $\boldsymbol{k}$ is now part of the structure, the subgroup $G$ of $K^{\times}$from Lemma 3.3 is definable. Now apply Lemma 3.4 and get a cross-section $s: \Gamma \rightarrow K^{\times}$such that $s(\Gamma)^{\dagger} \subseteq \boldsymbol{k}$. Take the additive map $c: \Gamma \rightarrow \boldsymbol{k}$ to be given by $c(\gamma)=s(\gamma)^{\dagger}$.

Proof of Theorem 圆. Let a monotone d-henselian valued field be given. Then it has a lift of its differential residue field, and fixing such a lift $\boldsymbol{k}$, it is a structure $((K, \boldsymbol{k}), \Gamma ; v)$ as above. Passing to an elementary extension, we can assume $((K, \boldsymbol{k}), \Gamma ; v)$ is $\aleph_{1}$-saturated. Then Theorem 3.5yields a cross-section $s: \Gamma \rightarrow K^{\times}$ and an additive map $c: \Gamma \rightarrow \boldsymbol{k}$ with $s(\gamma)^{\dagger}=c(\gamma)$ for all $\gamma \in \Gamma$. This in turn yields a Hahn field $\boldsymbol{k}\left(\left(t^{\Gamma}\right)\right)_{c}$ that is elementarily equivalent to $((K, \boldsymbol{k}), \Gamma ; v, s, c)$.

We can now prove Theorem 3

Proof of Theorem [3. Let $F$ be a monotone d-henselian valued field such that $v_{F}\left(C_{F}^{\times}\right)$ is pure in $\Gamma_{F}=v_{F}\left(F^{\times}\right)$. The valued differential field $F$ has a lift of its differential residue field, and fixing such a lift $\boldsymbol{k}_{F}$ we get the structure $\left(\left(F, \boldsymbol{k}_{F}\right), \Gamma_{F} ; v_{F}\right)$. Take 
an elementary extension $((K, \boldsymbol{k}), \Gamma ; v)$ of it that is $\aleph_{1}$-saturated. Then $\Delta:=v\left(C_{K}^{\times}\right)$ is pure in $v\left(K^{\times}\right)$. Since $\Delta$ is also $\aleph_{1}$-saturated (as an abelian group), we have a direct sum decomposition $\Gamma=\Delta \oplus \Gamma^{*}$ by [1, Corollary 3.3.37]. Since the valued subfield $C:=C_{K}$ of $K$ is $\aleph_{1}$-saturated, it has a cross-section $s_{C}: \Delta \rightarrow C^{\times}$. Theorem 3.5 yields a cross-section $\tilde{s}: \Gamma \rightarrow K^{\times}$of the valued field $K$ such that $\tilde{s}(\Gamma)^{\dagger} \subseteq \boldsymbol{k}$. By the definition of $\Delta$ we have $\tilde{s}(\gamma) \notin C$ for all $\gamma \in \Gamma \backslash \Delta$.

Let $s$ be the cross-section of the valued field $K$ that agrees with $s_{C}$ on $\Delta$ and with $\tilde{s}$ on $\Gamma^{*}$. Then $s(\gamma)^{\dagger} \in \boldsymbol{k}$ for all $\gamma \in \Gamma$, so we have an additive map $c: \Gamma \rightarrow \boldsymbol{k}$ given by $c(\gamma)=s(\gamma)^{\dagger}$. Moreover, for $\gamma \in \Gamma$,

$$
c(\gamma)=0 \Leftrightarrow s(\gamma)^{\prime}=0 \Leftrightarrow s(\gamma) \in C \Leftrightarrow \gamma \in \Delta .
$$

This gives $\operatorname{ker}(c)=v\left(C^{\times}\right)$, and thus $c(\Gamma) \cap \boldsymbol{k}^{\dagger}=\{0\}$ by Proposition 3.1. Since $\operatorname{ker}(c)$ is a pure subgroup of $\Gamma$ then so is $\Delta$. This in turn yields a Hahn field $\boldsymbol{k}\left(\left(t^{\Gamma}\right)\right)_{c}$ with the required properties that is elementarily equivalent to $((K, \boldsymbol{k}), \Gamma ; v, s, c)$.

\section{Eliminating the CROSS-SeCtion}

Note that every $\mathcal{K} \models \operatorname{Mo}(\ell, s, c)$ satisfies the sentences

(1) $\forall \gamma \forall \delta \quad c(\gamma+\delta)=c(\gamma)+c(\delta)$,

(2) $\forall \gamma \exists x \neq 0 \quad v(x)=\gamma \& x^{\dagger}=c(\gamma)$.

These sentences don't mention the cross-section $s$. Below we derive the analogue of Theorem 2.1 in the setting without a cross-section. Let $L_{2}^{-}$be the language $L_{2}$ with the symbol $s$ for the cross-section removed. Let $\operatorname{Mo}(\ell, c)$ be the $L_{2}^{-}$-theory whose models are the $L_{2}^{-}$-structures

$$
\mathcal{K}=(K, \Gamma ; v, c),
$$

where $K$ is a differential field equipped with a differential subfield $\boldsymbol{k}$ (singled out by a unary predicate symbol), $\Gamma$ is an ordered abelian group, $v: K^{\times} \rightarrow \Gamma=v\left(K^{\times}\right)$is a valuation that makes $K$ into a monotone valued differential field such that $\boldsymbol{k} \subseteq K$ is a lift of the differential residue field, and $c: \Gamma \rightarrow \boldsymbol{k}$ is such that the sentences (1) and (2) above are satisfied.

Lemma 4.1. Suppose $\mathcal{K}=(K, \Gamma ; v, c) \models \operatorname{Mo}(\ell, c)$ is d-henselian and $\aleph_{1}$-saturated. Then there is a cross-section $s: \Gamma \rightarrow K^{\times}$such that $s(\gamma)^{\dagger}=c(\gamma)$ for all $\gamma \in \Gamma$.

Proof. By (1) and (2) we have a definable subgroup $G:=\left\{x \in K^{\times}: x^{\dagger}=c(v(x))\right\}$ of $K^{\times}$with $v(G)=\Gamma$. Now, use Lemma 3.4 to get a cross section $s: \Gamma \rightarrow K^{\times}$with $s(\Gamma) \subseteq G$. This $s$ has the desired property.

Theorem 4.2. Suppose $\mathcal{K}=(K, \Gamma ; v, c) \models \operatorname{Mo}(\ell, c)$ is d-henselian. Then $\operatorname{Th}(\mathcal{K})$ is axiomatized by the following axiom schemes:

(1) $\operatorname{Mo}(\ell, c)$;

(2) the axioms for d-henselianity;

(3) $\operatorname{Th}(\boldsymbol{k}, \Gamma ; c)$ with $\boldsymbol{k}$ as differential field and $\Gamma$ as ordered abelian group.

Proof. Let any two $\aleph_{1}$-saturated models of the axioms in the theorem be given. By Lemma 4.1] we have in both models a cross-section that make these into models of $\operatorname{Mo}(\ell, s, c)$. It remains to appeal to Theorem 2.1 to conclude that these two models are elementarily equivalent. 
Before giving the proof of Corollary 1 from the introduction we note that any algebraic valued differential field extension of a monotone valued differential field is again monotone; see [1, Corollary 6.3.10].

Proof of Corollary 1. Let $K$ range over d-henselian monotone valued differential fields. As in [1, Proof of Corollary 8.0.2] we have a set $\Sigma_{n}$ of sentences in the language of valued differential fields, independent of $K$, such that $K \models \Sigma_{n}$ if and only if every valued differential field extension $L$ of $K$ with $[L: K]=n$ is dhenselian. Now by Theorem 2 we have $K \equiv \boldsymbol{k}\left(\left(t^{\Gamma}\right)\right)_{c}$ for a suitable differential field $\boldsymbol{k}$, ordered abelian group $\Gamma$, and additive map $c: \Gamma \rightarrow \boldsymbol{k}$. Every valued differential field extension $L$ of $\boldsymbol{k}\left(\left(t^{\Gamma}\right)\right)_{c}$ of finite degree is spherically complete as a valued field and so d-henselian by [1, Corollary 5.4.3 and Theorem 7.2.6]. Hence $\boldsymbol{k}\left(\left(t^{\Gamma}\right)\right)_{c}=\Sigma_{n}$ and thus $K \models \Sigma_{n}$, for all $n \geq 1$.

We now give an example of a monotone d-henselian field $F$ such that $v\left(C_{F}^{\times}\right)$is not pure in $v\left(F^{\times}\right)$. This elaborates on an example by the referee of a monotone henselian valued differential field $F$ for which $v\left(C_{F}^{\times}\right)$is not pure in $v\left(F^{\times}\right)$.

Let the additive map $c: \mathbb{Z} \rightarrow \mathbb{T}_{\log }$ be given by $c(1)=1$. With the usual derivation on $\mathbb{T}_{\log }$, this yields the (discretely) valued differential field $\boldsymbol{k}=\mathbb{T}_{\log }\left(\left(s^{\mathbb{Z}}\right)\right)_{c}$, with $s^{\prime}=s$. Since $\mathbb{T}_{\log }$ is linearly surjective, $\boldsymbol{k}$ is d-henselian field and thus linearly surjective. We now forget about the valuation of $\boldsymbol{k}$, consider it just as a differential field, and introduce $K:=\boldsymbol{k}\left(\left(t^{\mathbb{Z}}\right)\right)_{d}$ with the additive map $d: \mathbb{Z} \rightarrow \boldsymbol{k}$ given by $d(1)=0$, so $t^{\prime}=0$. Then $K$ is a monotone d-henselian field with $v\left(K^{\times}\right)=\mathbb{Z}$. Finally, let $F:=K(\sqrt{s t})$, which is naturally a valued differential field extension of $K$. Since $F$ is algebraic over $K$, it is monotone and d-henselian too, by Corollary 1 Clearly, $v\left(F^{\times}\right)=\frac{1}{2} \mathbb{Z}$. We claim that $v\left(C_{F}^{\times}\right)=\mathbb{Z}$ and so it is not pure in $v\left(F^{\times}\right)$. From $t^{\mathbb{Z}} \subseteq C_{F}$ we get $\mathbb{Z} \subseteq v\left(C_{F}^{\times}\right)$. For the reverse inclusion, let any element $a+b \sqrt{s t} \in C_{F}^{\times}$be given with $a, b \in K$, not both zero. Now,

$$
(a+b \sqrt{s t})^{\prime}=a^{\prime}+b^{\prime} \sqrt{s t}+b(\sqrt{s t})^{\prime}=a^{\prime}+b^{\prime} \sqrt{s t}+b(\sqrt{s t} / 2)=a^{\prime}+\left(b^{\prime}+b / 2\right) \sqrt{s t},
$$

so $a^{\prime}=0$ and $b^{\prime}+b / 2=0$. From $b^{\prime}=-b / 2$ we now derive $b=0$. (Then $a+b \sqrt{s t}=a \in C_{\boldsymbol{k}}\left(\left(t^{\mathbb{Z}}\right)\right)$, and thus $v(a+b \sqrt{s t}) \in \mathbb{Z}$, as claimed.) Let $k, l$ range over $\mathbb{Z}$. Towards a contradiction, suppose $b=\sum_{l \geq l_{0}} b_{l} t^{l}$ with all $b_{l} \in \boldsymbol{k}, l_{0} \in \mathbb{Z}, b_{l_{0}} \neq 0$.

Then $b^{\prime}=\sum_{l \geq l_{0}} b_{l}^{\prime} t^{l}$ and so the equality $b^{\prime}=-b / 2$ takes the form

$$
\sum_{l \geq l_{0}} b_{l}^{\prime} t^{l}=-\frac{1}{2} \sum_{l \geq l_{0}} b_{l} t^{l}=\sum_{l \geq l_{0}}-\frac{1}{2} b_{l} t^{l}
$$

Therefore $b_{l}^{\prime}=-b_{l} / 2$ for all $l \geq l_{0}$, in particular for $l=l_{0}$. Assume $b_{l_{0}}=\sum_{k \geq k_{0}} u_{k} s^{k}$, with all $u_{k} \in \mathbb{T}_{\log }$, and $k_{0} \in \mathbb{Z}, u_{k_{0}} \neq 0$. We have $b_{l_{0}}^{\prime}=\sum_{k \geq k_{0}}\left(u_{k}^{\prime}+k u_{k}\right) s^{k}$ and $-\frac{1}{2} b_{l_{0}}=\sum_{k \geq k_{0}}-\frac{1}{2} u_{k} s^{k}$. Thus $u_{k}^{\prime}+k u_{k}=-u_{k} / 2$ for all $k \geq k_{0}$. For $k=k_{0}$ we have $u_{k_{0}} \neq 0$, and so this gives $u_{k_{0}}^{\dagger}=-k_{0}-1 / 2$. However, this contradicts $\mathbb{T}_{\log }^{\dagger} \cap \mathbb{R}=\{0\}$ and hence the claim is proved.

On the other hand: 
Proposition 4.3. Let $F$ be a henselian valued differential field with algebraically closed or real closed residue field. Then $v\left(C_{F}^{\times}\right)$is pure in $v\left(F^{\times}\right)$.

Proof. Let $n \alpha=\beta$ with $\alpha \in v\left(F^{\times}\right), \beta \in v\left(C_{F}^{\times}\right), n \geq 1$; our job is to show that then $\alpha \in v\left(C_{F}^{\times}\right)$. Take $a \in F^{\times}$with $v(a)=\alpha$ and $b \in C_{F}^{\times}$with $v(b)=\beta$, so $v\left(b / a^{n}\right)=0$; if the residue field is real closed we also arrange that the residue class of $b / a^{n}$ is positive. Considering the polynomial $P(Y)=Y^{n}-\left(b / a^{n}\right) \in \mathcal{O}_{F}[Y]$, the henselianity of $F$ and the assumption on the residue field gives a zero $y \asymp 1$ in $F$ of $P$. Then $(a y)^{n}=b \in C_{F}^{\times}$, hence $a y \in C_{F}^{\times}$with $v(a y)=\alpha$.

A valued differential field with small derivation is said to be d-algebraically maximal if it has no proper immediate d-algebraic valued differential field extension. For monotone valued differential fields with linearly surjective differential residue field,

$$
\text { d-algebraically maximal } \Longrightarrow \text { d-henselian }
$$

by [1, Theorem 7.0.1]. By [1, Theorem 7.0.3], the converse holds in the case of few constants, but an example at the end of Section 7.4 of [1] shows that this converse fails for some d-henselian monotone valued differential field with many constants. Below we generalize this example as follows:

Corollary 4.4. Let $K$ be a d-henselian, monotone, valued differential field with $v\left(C^{\times}\right) \neq\{0\}$. Then some $L \equiv K$ is not d-algebraically maximal.

Proof. By Theorems 1 and 2 and Löwenheim-Skolem we can arrange $K=\boldsymbol{k}\left(\left(t^{\Gamma}\right)\right)_{c}$ where the differential field $\boldsymbol{k}$ and the ordered abelian group $\Gamma$ are countable and $c: \Gamma \rightarrow \boldsymbol{k}$ is additive. With $C:=C_{K}$, take $a \in C^{\times}$with $v a=\gamma_{0}>0$. Then $a=\sum_{\gamma \geq \gamma_{0}} a_{\gamma} t^{\gamma}$, with $\partial\left(a_{\gamma}\right)+c(\gamma) a_{\gamma}=0$ for all $\gamma$, in particular for $\gamma=\gamma_{0}$. Hence $\mathfrak{m}:=a_{\gamma_{0}} t^{\gamma_{0}} \in C$, and so all infinite sums $\sum_{n} q_{n} \mathfrak{m}^{n}$ with rational $q_{n}$ lie in $C$ as well. Thus $C$ is uncountable.

On the other hand, $\boldsymbol{k}\left(t^{\Gamma}\right)$ is countable and so by Löwenheim-Skolem we have a countable $L \prec K$ that contains $\boldsymbol{k}\left(t^{\Gamma}\right)$. Thus $K$ is an immediate extension of $L$ and we can take $a \in C \backslash L$. Then $L\langle a\rangle=L(a)$ is a proper immediate d-algebraic extension of $L$ and therefore $L$ is not d-algebraically maximal.

\section{Eliminating the Lift of the Differential RESidue FiELD}

In this section we drop the requirement of having a lift of the differential residue field in our structure and instead use a copy of the differential residue field. For this purpose we consider 3 -sorted structures

$$
\mathcal{K}=(K, \mathbf{k}, \Gamma ; \pi, v, c)
$$

where $K$ and $\mathbf{k}$ are differential fields, $\Gamma$ is an ordered abelian group, $v: K^{\times} \rightarrow \Gamma$ is a valuation which makes $K$ into a monotone valued differential field, $\pi: \mathcal{O} \rightarrow \mathbf{k}$ with $\mathcal{O}:=\mathcal{O}_{v}$ is a surjective differential ring morphism, $c: \Gamma \rightarrow \mathbf{k}$ is an additive map satisfying $\forall \gamma \exists x \neq 0 \quad\left[v(x)=\gamma \& \pi\left(x^{\dagger}\right)=c(\gamma)\right]$. We construe these $\mathcal{K}$ as $L_{3}$-structures for a natural 3-sorted language $L_{3}$ (with unary function symbols for $\pi, v$ and $c$ ). We have an obvious set $\operatorname{Mo}(c)$ of $L_{3}$-sentences whose models are exactly these $\mathcal{K}$. 
Lemma 5.1. Suppose $\mathcal{K}=(K, \mathbf{k}, \Gamma ; \pi, v, c) \models \operatorname{Mo}(c)$ is d-henselian and $\boldsymbol{k}$ is any lift of the differential residue field. Then $(K, \Gamma ; v, \iota \circ c) \models \operatorname{Mo}(l, c)$, where $\iota: \mathbf{k} \rightarrow \boldsymbol{k}$ is the inverse of the differential field isomorphism $\left.\pi\right|_{\boldsymbol{k}}: \boldsymbol{k} \rightarrow \mathbf{k}$.

Proof. We need to check the two conditions from the previous section. First of all $\iota \circ c$ is obviously additive. Fix $\gamma \in \Gamma$. There is an element $x \in K^{\times}$with $v(x)=\gamma$ and $\pi\left(x^{\dagger}\right)=c(\gamma)$. Let $a=(\iota \circ \pi)\left(x^{\dagger}\right)=(\iota \circ c)(\gamma)$. As $a \in \boldsymbol{k}$ and $\pi(a)=\pi\left(x^{\dagger}\right)$, we get $x^{\dagger}=a+\epsilon$ for some $\epsilon \prec 1$. By [1, Corollary 7.1.9] we have $\epsilon=(1+\delta)^{\dagger}$ for some $\delta \prec 1$ and thus

$$
(\iota \circ c)(\gamma)=a=x^{\dagger}-(1+\delta)^{\dagger}=\left(\frac{x}{1+\delta}\right)^{\dagger}, \text { and } v\left(\frac{x}{1+\delta}\right)=v(x)=\gamma .
$$

This completes the proof of the lemma.

Theorem 5.2. Suppose $\mathcal{K}=(K, \mathbf{k}, \Gamma ; \pi, v, c) \models \operatorname{Mo}(c)$ is d-henselian. Then $\operatorname{Th}(\mathcal{K})$ is axiomatized by the following axiom schemes:

(1) $\operatorname{Mo}(c)$;

(2) the axioms for d-henselianity;

(3) $\operatorname{Th}(\mathbf{k}, \Gamma ; c)$ with $\mathbf{k}$ as differential field and $\Gamma$ as ordered abelian group.

Proof. Let any two $\aleph_{1}$-saturated models $\mathcal{K}_{1}=\left(K_{1}, \mathbf{k}_{1}, \Gamma_{1} ; \pi_{1}, v_{1}, c_{1}\right)$ and $\mathcal{K}_{2}=$ $\left(K_{2}, \mathbf{k}_{2}, \Gamma_{2} ; \pi_{2}, v_{2}, c_{2}\right)$ of the axioms in the theorem be given. By Lemma 5.1 we have in both models lifts of the differential residue fields that make these into models of $\operatorname{Mo}(\ell, c)$. So $\operatorname{Th}\left(\mathbf{k}_{i}, \Gamma_{i} ; c_{i}\right)=\operatorname{Th}\left(\boldsymbol{k}_{i}, \Gamma_{i} ; \iota_{i} \circ c_{i}\right)$ where $\iota_{i}$ is the isomorphism between the differential residue field $\mathbf{k}_{i}$ and its lift $\boldsymbol{k}_{i}$ for $i=1,2$. It remains to appeal to Theorem 4.2 to conclude that these two models are elementarily equivalent.

\section{ACKNOWLEDGMENTS}

The author thanks Lou van den Dries for numerous discussions and comments on this paper. The author also thanks the referee for pointing out an error in the previous version of the paper and for making other helpful comments.

\section{REFERENCES}

[1] M. Aschenbrenner, L. van den Dries, J. van der Hoeven, Asymptotic Differential Algebra and Model Theory of Transseries, Annals of Math. Studies, number 195, to appear; arXiv: $1509.02588 \mathrm{v} 3$.

[2] R. Cohn, Solutions in the general solution, in: H. Bass, P. Cassidy, J. Kovacic (eds.), Contributions to Algebra, 117-128, Academic Press, New York, 1977.

[3] J. Ax and S. Kochen,Diophantine problems over local fields, III, Annals of Math. 83 (1966), 437-456.

[4] Ju. Ershov, On the elementary theory of maximal normed fields, Soviet Math. Dokl. 6 (1965), $1390-1393$.

[5] T. Scanlon, A model complete theory of valued d-fields, J. Symbolic Logic 65 (2000), 17581784 .

Department of Mathematics, University of Illinois at Urbana-Champaign, Urbana, IL 61801, U.S.A.

E-mail address: hakobya2@illinois.edu 